\title{
On the $P_{3}$-hull number of Kneser graphs
}

\author{
Luciano N. Grippo ${ }^{* \dagger * *} \quad$ Adrián Pastine ${ }^{\ddagger * *} \quad$ Pablo Torres ${ }^{\dagger}{ }^{\dagger * *}$ \\ Mario Valencia-Pabon $\mathbb{\Phi} * *^{\text {Juan C. Vera }}{ }^{\| * *}$
}

Submitted: Sep 27, 2020; Accepted: Jul 25, 2021; Published: Jul 30, 2021

(C) The authors. Released under the CC BY-ND license (International 4.0).

\begin{abstract}
This paper considers an infection spreading in a graph; a vertex gets infected if at least two of its neighbors are infected. The $P_{3}$-hull number is the minimum size of a vertex set that eventually infects the whole graph.

In the specific case of the Kneser graph $K(n, k)$, with $n \geqslant 2 k+1$, an infection spreading on the family of $k$-sets of an $n$-set is considered. A set is infected whenever two sets disjoint from it are infected. We compute the exact value of the $P_{3}$-hull number of $K(n, k)$ for $n>2 k+1$. For $n=2 k+1$, using graph homomorphisms from the Knesser graph to the Hypercube, we give lower and upper bounds.
\end{abstract}

Mathematics Subject Classifications: 05C76, 52A37, 05C85

\section{Introduction}

We only consider finite, simple, and undirected graphs. For a graph $G=(V, E)$, a graph convexity on $V$ is a collection $\mathcal{C}$ of subsets of $V$ such that $\varnothing, V \in \mathcal{C}$ and $\mathcal{C}$ is closed under intersections. The sets in $\mathcal{C}$ are called convex sets and the convex hull $H_{\mathcal{C}}(S)$ in $\mathcal{C}$ of a set $S$ of vertices of $G$ is the smallest set in $\mathcal{C}$ containing $S$ (see [7] and references therein). Some natural convexities in graphs are defined by a set $\mathcal{P}$ of paths in $G$, in a way that a set $S$ of vertices of $G$ is convex if and only if for every path $P: v_{0}, v_{1}, \ldots, v_{l} \in \mathcal{P}$ such that $v_{0}$ and $v_{l}$ belong to $S$, all vertices of $P$ belong to $S$ (cf. $[1,8]$ ). If we define $\mathcal{P}$ as the set of all shortest paths in $G$, we have the well-known geodetic convexity (see for example

\footnotetext{
*Instituto de Ciencias, Universidad Nacional de General Sarmiento, Argentina.

†Consejo Nacional de Investigaciones Científicas y Técnicas, Argentina.

¥Instituto de Matemática Aplicada San Luis, Universidad Nacional de San Luis, Argentina.

$\S$ Facultad de Ciencias Exactas, Ingeniería y Agrimensura, Universidad Nacional de Rosario, Argentina.

ILIPN, Université Sorbonne Paris Nord, France.

$\|$ Tilburg School of Economics and Management, The Netherlands.

**Email addresses: lgrippo@ungs.edu.ar (L. N. Grippo), agpastine@unsl.edu.ar (A. Pastine), ptorres@fceia.unr.edu.ar (P. Torres), valencia@lipn.univ-paris13.fr (M. Valencia-Pabon), j.c.veralizcano@tilburguniversity.edu (J. C. Vera).
} 
$[17,11,25])$. The monophonic convexity is defined by considering $\mathcal{P}$ as the set of all induced paths of $G[18,15]$.

If we let $\mathcal{P}$ be the set of all paths of $G$ with three vertices, we have the well-known $P_{3}$-convexity which will be studied in this paper. This convexity was introduced with the aim of modeling the spread of a disease in a grid [5]. Since then, many articles, in connection with this convexity, were published in the specialized literature (the reader is referred for instance to $[10,9,2,16,7])$.

Given a set $S \subseteq V$, the $P_{3}$-interval $I[S]$ of $S$ is formed by $S$, together with every vertex outside $S$ with at least two neighbors in $S$. If $I[S]=S$, then the set $S$ is $P_{3}$-convex. The $P_{3}$-convex hull $H_{\mathcal{C}}(S)$ of $S$ is the smallest $P_{3}$-convex set containing $S$. In what follows, we write $H(S)$ instead of $H_{\mathcal{C}}(S)$. The $P_{3}$-convex hull $H(S)$ can be formed from the sequence $I^{p}[S]$, where $p$ is a nonnegative integer, $I^{0}[S]=S, I^{1}[S]=I[S]$, and $I^{p}[S]=I\left[I^{p-1}[S]\right]$, for every $p \geqslant 2$. When for some $p \in \mathbb{N}$, we have $I^{q}[S]=I^{p}[S]$, for all $q \geqslant p$, then $I^{p}[S]$ is a $P_{3}$-convex set. If $H(S)=V(G)$ we say that $S$ is a $P_{3}$-hull set of $G$. The cardinality $h_{P_{3}}(G)$ of a minimum $P_{3}$-hull set in $G$ is called the $P_{3}$-hull number of $G$. Centeno et al. proved that, given a graph $G$ and an integer $k$, to decide whether the $P_{3}$-hull number of $\mathrm{G}$ is at most $k$ is an NP-complete problem [10]. Coelho et al. [14] proved that compute the $P_{3}$-hull number is an APX-hard problem even for bipartite graphs with maximum degree four. Moreover, Chen [12] shown that the $P_{3}$-hull number of a graph is hard to approximate within a ratio $O\left(2^{\log ^{1-\epsilon} n}\right)$, for any $\epsilon>0$, unless $\operatorname{NP} \subseteq \operatorname{DTIME}\left(n^{\text {polylog}(n)}\right)$. All these negative results motivate the study of the $P_{3}$-hull number on particular families of graphs.

In this paper we deal with the problem of computing the $P_{3}$-hull number of Kneser graphs $\mathrm{K}(n, k)$. Kneser graphs have a very nice structure. For an overview on this relevant family of graphs we refer the reader to [20]. Many graph theoretic parameters have been computed for Kneser graphs $\mathrm{K}(n, k)$. Some examples are the independence number [19], the chromatic number [22], the diameter [28].

The aim of this work is twofold, first to contribute to the knowledge of Kneser graphs; second to obtain new formulas for the hull number within a family of graphs having nice structure.

This article is organized as follows. In Section 2 we present some preliminaries definitions and concepts. Section 3 is devoted to our results. Finally, we give some concluding remarks in Section 4.

\section{Related work}

Infection problems appear in the literature under many different names and were studied by researches of various fields [13]. An infection problem already studied on Kneser graphs is zero forcing (see [6]). The zero forcing problem follows the infection rule where an infected vertex $v$ will infect one of its neighbors $w$ if all neighbors of $v$ except for $w$ are already infected. The zero forcing number of $G$ is the size of a smallest set $S$ of initially infected vertices that forces the whole graph to become infected. Another infection problem is the bootstrap percolation on a graph (see for example, $[4,3,23$, $24,26,27]$ and references therein): an infection spreads over the vertices of a connected 
graph $G$ following a deterministic spreading rule in such a way that an infected vertex will remain infected forever. Given a set $S \subseteq V(G)$ of initially infected vertices, we can build a sequence $S_{0}=S, S_{1}, S_{2}, \ldots$ in which $S_{i+1}$ is obtained from $S_{i}$ using such a spreading rule. Under the $r$-neighbor bootstrap percolation on a graph $G$, the spreading rule is a threshold rule in which $S_{i+1}$ is obtained from $S_{i}$ by adding to it the vertices of $G$ which have at least $r$ neighbors in $S_{i}$. The set $S_{0}$ is a percolating set of $G$ if there exists a $t$ such that $S_{t}=V(G)$. Let $t_{r}(S)$ be the minimum $t$ such that $S_{t}=V(G)$. The percolation time of $G$ is defined as $t_{r}(G)=\max \left\{t_{r}(S): S\right.$ percolates $\left.G\right\}$. Notice that this infection problem is related to graph convexities. In fact, the 2-neighbor bootstrap percolation problem on graphs is very close to the $P_{3}$-convexity on graphs. The 2 -neighbor bootstrap percolation problem has been studied by several authors. For example, the maximum percolation time of the 2-neighbor bootstrap percolation problem has been studied by Benevides et al. [4], Marcilon et al. [23] and Przykucki [26]. The smallest or largest size of a percolating set with a given property has been studied by Benevides et al. [3] and Morris [24]. Moreover, Przykucki [26] and Riedl [27] studied some problems concerning the size of 2-percolating sets. Notice that the problem of finding a minimum size 2-percolating set on a graph is equivalent to determining the $P_{3}$-hull number of such graph. As we have mentioned previously, the problem of computing the $P_{3}$-hull number of a graph is a very hard problem, even for bipartite graphs. Therefore, it is interesting to find infinite graph families where such parameter can be easily determined in polynomial time.

\section{Preliminaries}

Given a graph $G, N_{G}(u)$ stands for the neighborhood of $u$ in $G$. Let $A$ and $B$ be two sets. Given an integer $a$ such that $0 \leqslant a \leqslant|A|,\left(\begin{array}{l}A \\ a\end{array}\right)$ stands for the set whose elements are the $a$-element subsets of $A$, and $\left(\begin{array}{l}A \\ a\end{array}\right)\left(\begin{array}{l}B \\ b\end{array}\right)$ the set whose elements are the subsets of $A \cup B$ with $a$ elements in $A$ and $b$ elements in $B$. Notice that $\left(\begin{array}{l}A \\ 0\end{array}\right)=\{\varnothing\},\left(\begin{array}{l}A \\ 0\end{array}\right)\left(\begin{array}{l}B \\ b\end{array}\right)=\left(\begin{array}{l}B \\ b\end{array}\right)$, and $\left(\begin{array}{l}A \\ a\end{array}\right)\left(\begin{array}{l}B \\ 0\end{array}\right)=\left(\begin{array}{l}A \\ a\end{array}\right)$.

Let $n$ be a positive integer. We denote by $[n]$ the set $\{1, \cdots, n\}$. For positive integers $n$ and $k$ such that $n \geqslant 2 k$, the Kneser graph, denoted $\mathrm{K}(n, k)$, has as vertex set $\left(\begin{array}{c}{[n]} \\ k\end{array}\right)$ and two vertices are adjacent if they have empty intersection.

We introduce two more graphs in order to study the $P_{3}$-hull number of the Kneser graph $\mathrm{K}(2 k+1, k)$, the $n$-cube and middle levels graph. For any $n \in \mathbb{Z}^{+}$, the $n$-dimensional hypercube (or $n$-cube), denoted $Q_{n}$, is the graph in which the vertices are all binary $n$ tuples of length $n$ (i.e., the set $\{0,1\}^{n}$ ), and two vertices are adjacent if and only if they differ in exactly one position. For any $i \in\{0, \ldots, n\}$ we denote by $Q_{n}^{i}$ the $i$ th-layer of $Q_{n}$, that is, the subgraph of $Q_{n}$ induced by all the vertices having exactly $i$ ones.

The middle levels graph $M_{2 k+1}$ is the graph whose vertices are all $k$-element and all $(k+1)$-element subsets of $\{1,2, \ldots, 2 k+1\}$, with an edge between any pair of sets where one is a proper subset of the other. The name middle levels graph for $M_{2 k+1}$ comes from the fact that it is isomorphic to the subgraph of the hypercube $Q_{2 k+1}$ induced by all the vertices in the middle two layers $Q_{2 k+1}^{k}$ and $Q_{2 k+1}^{k+1}$. It is not difficult to see that $M_{2 k+1}$ is a 
bipartite connected graph of order $2\left(\begin{array}{c}2 k+1 \\ k\end{array}\right)$. Johnson and Kierstead [21] provide a natural 2-to-1 graph homomorphism $\phi$ from $M_{2 k+1}$ to $\mathrm{K}(2 k+1, k)$ defined by:

$$
\phi(X)= \begin{cases}X, & \text { if }|X|=k \\ \{1, \cdots, 2 k+1\} \backslash X, & \text { if }|X|=k+1\end{cases}
$$

\section{$3 \quad$ Hull number of Kneser graphs}

Let $k \geqslant 1$ and $n \geqslant 2 k+1$. For $i \in\{0, \ldots, k\}$, let $\mathcal{F}_{i}=\left(\begin{array}{c}{[k+1]} \\ i\end{array}\right)\left(\begin{array}{c}{[n] \backslash[k+1]} \\ k-i\end{array}\right)$. Then $\left\{\mathcal{F}_{i}: i=\right.$ $0, \ldots, k\}$ is a partition of the vertex set of $\mathrm{K}(n, k)$.

Lemma 1 . Let $k \geqslant 1$ and $n \geqslant 2 k+1$. Let $i, j \leqslant k$ be such that $i \leqslant j+1 \leqslant i+n-2 k$ and $(i, j) \notin\{(1,0),(3 k+1-n, k)\}$. Then, $\mathcal{F}_{i} \subseteq I\left[\mathcal{F}_{k-j}\right]$.

Proof. Let $0 \leqslant i, j \leqslant k$. Any vertex in $\mathcal{F}_{i}$ has exactly $d_{i, j}:=\left(\begin{array}{c}k+1-i \\ k-j\end{array}\right)\left(\begin{array}{c}n-2 k+i-1 \\ j\end{array}\right)$ neighbors in $\mathcal{F}_{k-j}$. Thus $\mathcal{F}_{i} \subseteq I\left[\mathcal{F}_{k-j}\right]$ if and only if $d_{i, j} \geqslant 2$. As $d_{i, j} \geqslant 0$ we analyze when it is equal to zero or one. Notice that $d_{i, j}=0$ if and only if $j+1<i$ or $j+1>i+n-2 k$. Also, $d_{i, j}=1$ if and only if $\left(\begin{array}{c}k+1-i \\ k-j\end{array}\right)=1$ and $\left(\begin{array}{c}n-2 k+i-1 \\ j\end{array}\right)=1$. That is when $j=k$ or $j+1=i$, and $j=0$ or $j+1=i+n-2 k$.

Lemma 2. Let $k \geqslant 1$ and $n \geqslant 2 k+1$. Then $\mathcal{F}_{1}$ is a hull set of $\mathrm{K}(n, k)$.

Proof. First we show by induction that $\mathcal{F}_{t} \cup \mathcal{F}_{k-t} \subset H\left(\mathcal{F}_{1}\right)$ for $t=1, \ldots,\lfloor k / 2\rfloor$. To do this notice that taking $i=j=k-1$ in Lemma 1 we obtain the base case $t=1$. Now assume the statement is true for $t \geqslant 1$. Taking $i=t+1$ and $j=t$ in Lemma 1 we obtain $\mathcal{F}_{t+1} \subset I\left[\mathcal{F}_{k-t}\right] \subset H\left(\mathcal{F}_{1}\right)$. Also, taking $i=j=k-t-1$ we obtain $\mathcal{F}_{k-t-1} \subset I\left[\mathcal{F}_{t+1}\right]$ completing the induction. To finish the proof, notice that taking $i=k$ and $j=k-1$ in Lemma 1 we obtain $\mathcal{F}_{k} \subset I\left[\mathcal{F}_{1}\right]$ and taking $i=j=0$ in Lemma 1 we obtain $\mathcal{F}_{0} \subset$ $I\left[\mathcal{F}_{k}\right]$.

Theorem 3. Let $k \geqslant 1$ and $n \geqslant 2 k+3$. Then $h_{P_{3}}(\mathrm{~K}(n, k))=2$.

Proof. Let $A_{1}=[k]$ and $A_{2}=[k+1] \backslash\{k\}$ and define $\mathcal{S}=\left\{A_{1}, A_{2}\right\}$. We will show that $\left\{A_{1}, A_{2}\right\}$ is a $P_{3}$-hull set of $\mathrm{K}(n, k)$.

Notice that $A_{1}$ and $A_{2}$ are neighbors of all the vertices in $\mathcal{F}_{0}$. Hence $\mathcal{F}_{0} \subset H(\mathcal{S})$. Taking $i=j=k$ in Lemma 1 we obtain $\mathcal{F}_{k} \subset I\left[\mathcal{F}_{0}\right] \subset H(\mathcal{S})$. Taking $i=k-1$ and $j=k$ in Lemma 1 we obtain $\mathcal{F}_{k-1} \subset I\left[\mathcal{F}_{0}\right] \subset H(\mathcal{S})$. Also, taking $i=j=1$ we obtain $\mathcal{F}_{1} \subset I\left[\mathcal{F}_{k-1}\right] \subset H(\mathcal{S})$. The statement follows by Lemma 2 .

Theorem 4. $h_{P_{3}}(\mathrm{~K}(2 k+2, k))=3$, for every $k \geqslant 3$.

Proof. First, we will prove that $h_{P_{3}}(\mathrm{~K}(2 k+2, k))>2$. Let $\mathcal{S}=\left\{S_{1}, S_{2}\right\} \subseteq \mathrm{K}(2 k+2, k)$ and let $A=S_{1} \cup S_{2}$. We split the proof into the only two possible cases for $\left|S_{1} \cap S_{2}\right|$.

Case 1: $\left|S_{1} \cap S_{2}\right|=k-1$. 
Since $|A|=k+1$, each vertex in $\left(\begin{array}{l}\bar{A} \\ k\end{array}\right)$ is adjacent to $S_{1}$ and $S_{2}$ and thus $\left(\begin{array}{l}\bar{A} \\ k\end{array}\right) \subseteq I[\mathcal{S}]$. Symmetrically, since $|\bar{A}|=k+1$ and $\left(\begin{array}{l}\bar{A} \\ k\end{array}\right) \subseteq I[\mathcal{S}]$, we conclude that $\left(\begin{array}{l}A \\ k\end{array}\right) \subseteq I^{2}[\mathcal{S}]$. Let $C$ be any vertex in $\mathrm{K}(2 k+2, k)$. Since $|C|=k \geqslant 3$, either $|C \cap A| \geqslant 2$ or $|C \cap \bar{A}| \geqslant 2$. Assume, without loosing generality, that $|C \cap A| \geqslant 2$. If $C \notin\left(\begin{array}{l}A \\ k\end{array}\right) \cup\left(\begin{array}{l}\bar{A} \\ k\end{array}\right)$, then $|C \cap \bar{A}| \geqslant 1$. Hence $C$ has no neighbors in $\left(\begin{array}{l}A \\ k\end{array}\right)$ and it has at most one neighbor in $\left(\begin{array}{l}\bar{A} \\ k\end{array}\right)$ which implies that $C \notin H\left(\left(\begin{array}{l}A \\ k\end{array}\right) \cup\left(\begin{array}{l}\bar{A} \\ k\end{array}\right)\right)$. Therefore, $H(\mathcal{S})=I^{2}[\mathcal{S}]=\left(\begin{array}{l}A \\ k\end{array}\right) \cup\left(\begin{array}{l}\bar{A} \\ k\end{array}\right)$.

Case 2: $\left|S_{1} \cap S_{2}\right|=k-2$.

Let $A=S_{1} \cup S_{2}$. Hence, $|A|=k+2$ and $|\bar{A}|=k$. Thus, $\left(\begin{array}{l}\bar{A} \\ k\end{array}\right)=\{\bar{A}\}$ and $I[\mathcal{S}]=$ $\left\{\bar{A}, S_{1}, S_{2}\right\}$. In addition, for each $i \in\{1,2\}, C \cap S_{i} \neq \varnothing$ for every $C \in\left(\begin{array}{l}A \\ k\end{array}\right)$. Therefore, $H(\mathcal{S})=\left\{S_{1}, S_{2}, \bar{A}\right\}$.

Since in both cases $H\left(\left\{S_{1}, S_{2}\right\}\right)$ is properly contained in $\mathrm{K}(2 k+2, k)$, we conclude that $h_{P_{3}}(\mathrm{~K}(2 k+2, k)) \geqslant 3$.

To show $h_{P_{3}}(\mathrm{~K}(2 k+2, k)) \leqslant 3$, let $\mathcal{S}=\left\{A_{1}, A_{2}, A_{3}\right\}$, where $A_{1}=[k], A_{2}=[k+1] \backslash\{k\}$ and $A_{3}=\{3, \ldots, k+2\}$. We will prove that $\mathcal{S}$ is a hull set of $\mathrm{K}(2 k+2, k)$. As in the proof of Theorem $3, A_{1}$ and $A_{2}$ are neighbors of all the vertices in $\mathcal{F}_{0}$ and hence $\mathcal{F}_{0} \subset$ $I^{1}\left(\left\{A_{1}, A_{2}\right\}\right)$. Taking $i=j=k$ in Lemma 1 we obtain $\mathcal{F}_{k} \subseteq H\left[\left\{A_{1}, A_{2}\right\}\right]$. We have, then, $\left\{A_{1}, A_{2}\right\} \subset \mathcal{F}_{0} \cup \mathcal{F}_{k} \subseteq H\left[\left\{A_{1}, A_{2}\right\}\right]$. It is not difficult to see that $\mathcal{F}_{0} \cup \mathcal{F}_{k}=H\left[\left\{A_{1}, A_{2}\right\}\right]$, as $I\left[\mathcal{F}_{0} \cup \mathcal{F}_{k}\right]=\mathcal{F}_{0} \cup \mathcal{F}_{k}$. Indeed, for any $B \in \mathcal{F}_{j}$ with $0<j<k$ we have $B$ connected to $\mathcal{F}_{0} \cup \mathcal{F}_{k}$ only when $j=1$ or $j=k-1$. If $j=1, B$ has exactly one neighbor in $\mathcal{F}_{k}$ and none in $\mathcal{F}_{0}$. Similarly, when $j=k-1, B$ has exactly one neighbor in $\mathcal{F}_{0}$ and none in $\mathcal{F}_{k}$.

Let $\mathcal{S}_{1}=\left(\begin{array}{c}\{1,2\} \\ 1\end{array}\right)\left(\begin{array}{c}{[2 k+2] \backslash[k+2]} \\ k-1\end{array}\right)$. We have $\mathcal{S}_{1}=N\left(A_{3}\right) \cap \mathcal{F}_{1}$. As $\mathcal{F}_{1} \subset N\left(H\left(\left\{A_{1}, A_{2}\right\}\right)\right)$ and $A_{3} \notin H\left(\left\{A_{1}, A_{2}\right\}\right)$ we have $\mathcal{S}_{1} \subset H(\mathcal{S})$. Now let $\mathcal{S}_{2}=\left\{A \in \mathcal{F}_{k-1}: A \cap\{1,2\}=1\right\}$. Every element in $\mathcal{S}_{2}$ has a neighbor in $\mathcal{S}_{1}$; to see this, let $a$ be the only element in $A \cap\{1,2\}$ and let $b$ be the only element in $A \backslash[k+1]$. Then, there exists $Y \subseteq\{a, k+3, \cdots, 2 k+2\} \backslash\{b\}$ with $Y \in N(A) \cap \mathcal{S}_{1}$. Also, $\mathcal{S}_{2} \subset \mathcal{F}_{k-1} \subset N\left(\mathcal{F}_{0}\right)$. As $\mathcal{F}_{0} \cap \mathcal{S}_{1} \subset \mathcal{F}_{0} \cap \mathcal{F}_{1}=\varnothing$, we have $\mathcal{S}_{2} \subset H(\mathcal{S})$.

Now we claim that $\mathcal{F}_{1} \subset N\left(\mathcal{S}_{2}\right)$. This implies that $\mathcal{F}_{1} \subset H(\mathcal{S})$, as $\mathcal{S}_{2} \cap \mathcal{F}_{k} \subset$ $\mathcal{F}_{k-1} \cap \mathcal{F}_{k}=\varnothing$ and $\mathcal{F}_{1} \subset N\left(\mathcal{F}_{k}\right)$. Notice that by Lemma 2 we obtain $H(\mathcal{S})=\mathrm{K}(2 k+1, k)$. To show $\mathcal{F}_{1} \subset N\left(\mathcal{S}_{2}\right)$, let $A \in \mathcal{F}_{1}$ and let $c \in A \cap[k+1]$. If $c \in\{1,2\}$, let $a$ be the integer in $\{1,2\} \backslash\{c\}$ and let $X$ be any $(k-2)$-set in $\left(\begin{array}{c}{[k+1] \backslash\{1,2\}} \\ k-2\end{array}\right)$. Otherwise, let $a=1$ and let $X$ be the only $(k-2)$-set in $[k+1] \backslash\{1,2, c\}$. Let $b \notin A \backslash[k+1]$. Then, $Y=\{a\} \cup X \cup\{b\}$ is a vertex in $\mathcal{S}_{2}$ having $A$ as a neighbor. Therefore, $Y \in N(A) \cap \mathcal{S}_{2}$ and so, $A \in N\left(\mathcal{S}_{2}\right)$.

Remark 5. $h_{P_{3}}(\mathrm{~K}(6,2))=2$.

Proof. Let $A=\{1,2,3\}$. Let $S_{i}=A \backslash\{i\}$ for each $i \in\{1,2\}$. Since $C \cap S_{i}=\varnothing$, for every $C \in\left(\begin{array}{c}\bar{A} \\ 2\end{array}\right)$ and for each $i \in\{1,2\},\left(\begin{array}{c}\bar{A} \\ 2\end{array}\right) \subseteq H\left(\left\{S_{1}, S_{2}\right\}\right)$. Hence $\left(\begin{array}{c}A \\ 2\end{array}\right) \subseteq H\left(\left\{S_{1}, S_{2}\right\}\right)$. If $C \notin\left(\begin{array}{l}A \\ 2\end{array}\right) \cup\left(\begin{array}{c}\bar{A} \\ 2\end{array}\right)$, then $|C \cap A|=|C \cap \bar{A}|=1$. Hence $C$ is adjacent to $A \backslash C \in\left(\begin{array}{l}A \\ 2\end{array}\right)$ and $\bar{A} \backslash C \in\left(\begin{array}{c}\bar{A} \\ 2\end{array}\right)$ and thus $C \in H\left(\left\{S_{1}, S_{2}\right\}\right)$. Therefore, $\left\{S_{1}, S_{2}\right\}$ is a hull set of $\mathrm{K}(6,2)$. 
Theorem 6. $h_{P_{3}}(\mathrm{~K}(2 k+1, k)) \leqslant k^{2}+k$.

Proof. From Lemma 2, we have $h_{P_{3}}(\mathrm{~K}(2 k+1, k)) \leqslant\left|\mathcal{F}_{1}\right|=k^{2}+k$.

\subsection{Preservation of $\boldsymbol{P}_{3}$ convexity under homomorphisms and its inverses}

Let $G=(V, E)$ a graph. For any vertex $u \in V$, let $N_{G}(u)$ denote the subset of neighbor vertices of $u$ in $G$, that is, the set $\{v \in V: u v \in E\}$. Let $G_{1}=\left(V_{1}, E_{1}\right)$ and $G_{2}=\left(V_{2}, E_{2}\right)$ be graphs. A graph homomorphism between graphs $G_{1}$ and $G_{2}$, denoted by $\phi: G_{1} \rightarrow G_{2}$, is a mapping $\phi$ from $V\left(G_{1}\right)$ to $V\left(G_{2}\right)$ such that $\phi(u)$ and $\phi(v)$ are adjacent in $G_{2}$ whenever $u$ and $v$ are adjacent in $G_{1}$. A graph homomorphism $\phi: G_{1} \rightarrow G_{2}$ is called locally bijective if for all $u \in G_{1}$ the restriction of $\phi$ to $N_{G_{1}}(u)$ is a bijection between $N_{G_{1}}(u)$ and $N_{G_{2}}(\phi(u))$.

Lemma 7. Let $\phi: G_{1} \rightarrow G_{2}$ be a locally bijective graph homomorphism. Let $S \subseteq G_{2}$. Then, $\phi^{-1}(H(S)) \subseteq H\left(\phi^{-1}(S)\right)$.

Proof. We prove by induction on $i$ that $\phi^{-1}\left(I^{i}[S]\right) \subseteq I^{i}\left[\phi^{-1}(S)\right]$ for all $i \geqslant 0$. In the base case $i=0$ we actually have equality. Now assume the statement is true for $i>0$. Let $u \in \phi^{-1}\left[I^{i+1}(S)\right]$, that is $\phi(u) \in I^{i+1}(S)$. If $\phi(u) \in I^{i}[S]$, then by induction $u \in$ $I^{i}\left[\phi^{-1}(S)\right] \subseteq I^{i+1}\left[\phi^{-1}(S)\right]$. Thus, assume $\phi(u) \notin I^{i}[S]$. Then there are two neighbors $v$ and $w$ of $\phi(u)$ in $I^{i}(S)$. By assumption, $\phi^{-1}(v) \cup \phi^{-1}(w) \subseteq I^{i}\left[\phi^{-1}(S)\right]$. As $\phi$ is locally bijective, $N_{G_{1}}(u) \cap \phi^{-1}(v)=\left\{v^{\prime}\right\}$ and $N_{G_{1}}(u) \cap \phi^{-1}(w)=\left\{w^{\prime}\right\}$, for some $v^{\prime}, w^{\prime} \in H$. As $v^{\prime}, w^{\prime} \in I^{i}\left[\phi^{-1}(S)\right]$ we have $u \in I^{i+1}\left[\phi^{-1}(S)\right]$.

Lemma 8. Let $\phi: G_{1} \rightarrow G_{2}$ be a locally bijective graph homomorphism. Let $S \subset G_{1}$. Then, $\phi(H(S)) \subseteq H(\phi(S))$.

Proof. We prove by induction on $i$ that $\phi\left(I^{i}[S]\right) \subseteq I^{i}[\phi(S)]$ for all $i \geqslant 0$. In the base case $i=0$ we actually have equality. Now assume the statement is true for $i>0$. Let $u \in I^{i+1}(S)$, we want to show $\phi(u) \in I^{i+1}[\phi(S)]$. If $u \in I^{i}[S]$, then by induction $\phi(u) \in I^{i}[\phi(S)] \subseteq I^{i+1}[\phi(S)]$. Thus, assume $u \notin I^{i}[S]$. Then $\left|N_{G_{1}}(u) \cap I^{i}[S]\right| \geqslant 2$. As $\phi$ is locally bijective, $\left|N_{G_{2}}(\phi(u)) \cap \phi\left(I^{i}[S]\right)\right| \geqslant 2$ also, and thus $u \in I^{i+1}[S]$.

Theorem 9. Let $\phi: G_{1} \rightarrow G_{2}$ be a surjective, locally bijective graph homomorphism. Then $h_{P_{3}}\left(G_{2}\right) \leqslant h_{P_{3}}\left(G_{1}\right) \leqslant \max \left\{\left|\phi^{-1}(u)\right|: u \in G_{2}\right\} h_{P_{3}}\left(G_{2}\right)$.

Proof. Let $S_{1}$ be hull set for $G_{1}$. From Lemma 8 we obtain $H\left(\phi\left(S_{1}\right)\right) \supseteq \phi\left(H\left(S_{1}\right)\right)=$ $\phi\left(G_{1}\right)=G_{2}$. Thus $h_{P_{3}}\left(G_{1}\right)=\left|S_{1}\right| \geqslant\left|\phi\left(S_{1}\right)\right| \geqslant h_{P_{3}}\left(G_{2}\right)$. Let $S_{2}$ be hull set for $G_{2}$. From Lemma $7, H\left(\phi^{-1}\left(S_{2}\right)\right) \supseteq \phi^{-1}\left(H\left(S_{2}\right)\right)=\phi^{-1}\left(G_{2}\right)=G_{1}$. Thus $h_{P_{3}}\left(G_{1}\right) \leqslant\left|\phi^{-1}\left(S_{2}\right)\right| \leqslant$ $\max \left\{\left|\phi^{-1}(u)\right|: u \in G_{2}\right\}\left|S_{2}\right|=\max \left\{\left|\phi^{-1}(u)\right|: u \in G_{2}\right\} h_{P_{3}}\left(G_{2}\right)$.

Corollary 10. Let $k \geqslant 1$ be an integer. Then, $h_{P_{3}}(\mathrm{~K}(2 k+1, k)) \leqslant h_{P_{3}}\left(M_{2 k+1}\right) \leqslant$ $2 h_{P_{3}}(\mathrm{~K}(2 k+1, k))$.

Proof. The result follows from Theorem 9 by noticing that the 2-to-1 graph homomorphism from $M_{2 k+1}$ to $\mathrm{K}(2 k+1, k)$ defined at the end of Section 2 is surjective and locally bijective. 


\subsection{Lower bound of $h_{P_{3}}(\mathrm{~K}(2 k+1, k))$}

In order to deduce a lower bound for $h_{P_{3}}(\mathrm{~K}(2 k+1, k))$, we need the following preliminary results.

Lemma 11. Let $n>1$ and $1 \leqslant i \leqslant n-1$ be integers. Let $S$ be the set of vertices in the ith-layer $Q_{n}^{i}$ of the hypercube $Q_{n}$. Then, $S$ is a $P_{3}$-hull set of $Q_{n}$.

Proof. Let $x=\left(x_{1}, \cdots, x_{n}\right)$ be any vertex in $Q_{n}^{i-1}$. Clearly, there exist two coordinates $x_{p}, x_{q}$ in $x$, with $1 \leqslant p<q \leqslant n$, such that $x_{p}=x_{q}=0$. The vertices $y=$ $\left(x_{1}, \cdots, x_{p-1}, 1, x_{p+1}, \cdots, x_{n}\right)$ and $z=\left(x_{1}, \cdots, x_{q-1}, 1, x_{q+1}, \cdots, x_{n}\right)$ are vertices in $S$ adjacent to $x$. In the same way, for any vertex $w$ in $Q_{n}^{i+1}$ we can pick two different coordinates $w_{p}$ and $w_{q}$ such that $w_{p}=w_{q}=1$. Then we can find two vertices $u$ and $v$ in $S$ adjacent to $w$, where $u$ (resp. $v$ ) is equal to $w$ except in the $p$ th (resp. qth) coordinate which is equal to 0 . Thus, $w$ has at least two neighbors in $S$. As this property holds for any $1 \leqslant i \leqslant n-1$ then, we conclude that $S$ is a $P_{3}$-hull set of the hypercube $Q_{n}$.

Concerning with the $P_{3}$-hull number of the $n$-dimensional hypercube $Q_{n}$, the following result has been obtained recently by Brešar and Valencia-Pabon [7].

Theorem 12 ([7]). For any $n \geqslant 1, h_{P_{3}}\left(Q_{n}\right)=\left\lceil\frac{n}{2}\right\rceil+1$.

Lemma 13. Let $k \geqslant 1$ be an integer. Then, $h_{P_{3}}\left(M_{2 k+1}\right) \geqslant k+2$.

Proof. Let $S$ be a $P_{3}$-hull set of $M_{2 k+1}$. For any vertex $w \in S$ let $\tilde{w}$ be a vertex in the hypercube $Q_{2 k+1}$ such that $\tilde{w}_{j}=1$ if $j \in w$, and $\tilde{w}_{j}=0$ otherwise, for $1 \leqslant j \leqslant 2 k+1$. As $M_{2 k+1}$ is isomorphic to the subgraph of $Q_{2 k+1}$ induced by the vertices in the two middle layers $Q_{2 k+1}^{k}$ and $Q_{2 k+1}^{k+1}$ then, by Lemma 11, the set $S^{\prime}=\{\tilde{w}: w \in S\}$ is a $P_{3}$-hull set of $Q_{2 k+1}$. Therefore, by Theorem $12,\left|S^{\prime}\right| \geqslant\left\lceil\frac{2 k+1}{2}\right\rceil+1=k+2$.

Finally, by Lemma 13 and Corollary 10, we have the following theorem.

Theorem 14. Let $k \geqslant 1$ be an integer. Then, $h_{P_{3}}(K(2 k+1, k)) \geqslant\left\lceil\frac{k}{2}\right\rceil+1$.

\section{Discussion}

Corollary 10 gives an upper bound for the $P_{3}$-hull number of $M_{2 k+1}$ in terms of the $P_{3}$-hull number of $\mathrm{K}(2 k+1, k)$. Exact values for $h_{P_{3}}(\mathrm{~K}(2 k+1, k))$ and $h_{P_{3}}\left(M_{2 k+1}\right)$, calculated with the aid of a computer, are shown in Table 1.

So we have the following conjecture.

Conjecture 15. $\left\lceil\frac{h_{P_{3}}\left(M_{2 k+1}\right)}{2}\right\rceil=h_{P_{3}}(\mathrm{~K}(2 k+1, k))$, for any integer $k \geqslant 1$.

The lower bound for the $P_{3}$-hull number of $\mathrm{K}(2 k+1, k)$ obtained in Theorem 14 seems to be far from being tight. In addition to results given in Table 1, we also have computational evidence showing that $h_{P_{3}}(\mathrm{~K}(2 k+1, k))$ is at most equal to 11,16 and 23 for $k=5,6$ and 7 , respectively. Notice that $h_{P_{3}}(\mathrm{~K}(2 k+1, k))$ seems to be equal to $\frac{k(k-1)}{2}+c$, being $c$ a constant, with $c \leqslant 2$. So we have the following conjecture.

Conjecture 16. $h_{P_{3}}(\mathrm{~K}(2 k+1, k))=\Theta\left(k^{2}\right)$. 


\begin{tabular}{|c|c|c|}
\hline$k$ & $h_{P_{3}}(\mathrm{~K}(2 k+1, k))$ & $h_{P_{3}}\left(M_{2 k+1}\right)$ \\
\hline 1 & 2 & 3 \\
\hline 2 & 3 & 6 \\
\hline 3 & 5 & 9 \\
\hline 4 & 8 & $\leqslant 15$ \\
\hline
\end{tabular}

Table 1: Some exact results.

\section{Acknowledgements}

The first four authors acknowledge partial support of LIA SINFIN/INFINIS (CNRSCONICET-UBA, France-Argentina) and partial support of Regional Program MATHAMSUD MATH190013 (France - Argentina - Chile). The first author acknowledges partial support of ANPCyT PICT 2017-1315.

\section{References}

[1] H. J. Bandelt. Graphs with intrinsic $s_{3}$ convexities. J. Graph Theory, 13(2):215-228, 1989.

[2] R. M. Barbosa, C. E. M. M., M. C. Dourado, D. Rautenbach, and J. L. Szwarcfiter. On the carathéodory number for the convexity of paths of order three. SIAM J. on Discrete Math., 26:929-939, 2012.

[3] F. Benevides and M. Przykucki. On slowly percolating sets of minimal size in bootstrap percolation. Electron J. Combin., 20(2), 2013, \#P46.

[4] F. Benevides, C. V., M. C. Dourado, R. M. Sampaio, and A. Silva. The maximum time of a 2-neighbour bootstrap percolation: Algorithmic aspects. European J. Combin., 48:88-99, 2015.

[5] B. Bollobás. The Art of Mathematics: Cofee Time in Memphis. Cambridge University Press, 2006.

[6] B. Brešar, T. Kos, and P. D. Torres. Grundy domination and zero forcing in Kneser graphs. Ars Math. Contemp., 17(2):419-430, 2019.

[7] B. Bresăr and M. Valencia-Pabon. On the $P_{3}$-hull number of Hamming graphs. Discrete Appl. Math., 282:48-52, 2020.

[8] J. Calder. Some elementary properties of interval convexities. J. London Math. Soc., 3:422-428, 1971.

[9] C. C. Centeno, S. Dantas, M. C. Dourado, D. Rautenbach, and J. L. Szwarcfiter. Convex partitions of graphs induced by paths of order three. Discrete Math. and Theoret. Comput. Sci., 12(5):175-184, 2010.

[10] C. C. Centeno, L. D. Penso, D. Rautenbach, and V. G. Pereira de Sá. Geodetic number versus hull number in $P_{3}$-convexity. SIAM J. on Discrete Math., 27(2):717$731,2013$. 
[11] M. Changat, H. M. Mulder, and G. Sierksma. Convexities related to path properties on graphs. Discrete Math., 290(2-3):117-131, 2005.

[12] N. Chen. On the approximability of influence in social networks. SIAM J. on Discrete Math., 23(3):1400-1415, 2009.

[13] M. Chopin. Optimization problems with propagation in graphs: parameterized complexity and approximation. PhD thesis, Université Paris-Dauphine, 2013.

[14] E. M. M. Cohelo, M. C. Dourado, and R. M. Sampaio. Inapproximability results for graph convexity parameters. Theoret. Comput. Sci., 600:49-58, 2015.

[15] M. C. Dourado, F. Protti, and J. L. Szwarcfiter. Complexity results related to monophonic convexity. Discrete Appl. Math., 158(12):1268-1274, 2010.

[16] M. C. Dourado, D. Rautenbach, V. Fernandes dos Santos, P. M. Schäfer, and J. L. Szwarcfiter. An upper bound on the $P_{3}$-Radon number. Discrete Math., 312:24332437, 2012.

[17] P. Duchet. Convexity in combinatorial structures. In Proceedings of the 14 th winter school on abstract analysis (Srní, 1986), number 14, pages 261-293, 1987.

[18] P. Duchet. Convex sets in graphs, ii. minimal path convexity. J. Comb. Theory B., 44(3):307-316, 1988.

[19] P. Erdős, C. Ko, and R. Rado. Intersection theorems for systems of finite sets. Quart. J. Math. Oxford Ser. (2), 12:313-320, 1961.

[20] C. Godsil and G. Royle. Algebraic graph theory. Springer, 2001.

[21] J. R. Johnson and H. A. Kierstead. Explicit 2-factorisations of the odd graph. Order, 21:19-27, 2004.

[22] L. Lovász. Kneser's conjecture, chromatic number, and homotopy. J. Combin. Theory Ser. A, 25(3):319-324, 1978.

[23] T. Marcilon and R. Sampaio. The maximum time of 2-neighbor bootstrap percolation: complexity results. Theoret. Comput. Sci., 708:1-17, 2018.

[24] R. Morris. Minimal percolating sets in bootstrap percolation. Electron J. Combin., 16(1), 2009, \#R2.

[25] I. M. Pelayo. Geodesic convexity in graphs. Springer, 2013.

[26] M. Przykucki. Maximal percolation time in hypercubes under 2-bootstrap percolation. Electron J. Combin., 19(2), 2012, \#P41.

[27] E. Riedl. Largest minimal percolating sets in hypercubes under 2-bootstrap percolation. Electron J. Combin., 17, 2010, \#R80.

[28] M. Valencia-Pabon and J.-C. Vera. On the diameter of Kneser graphs. Discrete Math., 305(1-3):383-385, 2005. 